\title{
Health care resource use in patients of the Australian Transition Care Program
}

\section{Key Question Summary}

1. What is known about the topic?

A majority of health care costs occur in older age

2. What does this paper add?

Hospital costs - both initial and re-admissions - constitute the major contributor to health care costs in transition care recipients. Orthopaedic conditions are the most expensive to treat and neurological conditions are the most variable.

3. What are the implications for practitioners?

Reducing the length of hospitalisation and reducing re-admissions for older frail people is a key economic concern for health services. Services such as the Transition Care Program aim to do both; however, the evidence that this is effective is limited. Streamlining referrals to Transition Care to enable earlier access and involving the Transition Care provider in readmission decisions may help reduce health care costs in the future. 


\section{Abstract}

\section{Objective:}

This paper aims to describe, from the perspective of the health care funder, the cost components of the Australian Transition Care Program. The paper also describes the health care resource use and costs for a group of Transition Care patients over a six month period.

\section{Methods:}

A prospective cohort observational study of 351 consenting patients entering communitybased Transition Care at six sites in two states in Australia was conducted from November 2009 to September 2010. Patients were followed up at six months post admission to the Transition Care Program to ascertain current living status and hospital readmissions over the follow-up period. Cost data were collected by transition care teams and from hospital and Medicare records.

\section{Results:}

The Transition Care Program provides a range of services with most costs attributed to provision of personal care support, case management, physiotherapy and occupational therapy. The majority of health care costs up to six months post transition care admission were incurred from the hospital admission leading to transition care (mean cost \$39,516) and from re-admissions to hospital (mean cost $\$ 7,038$ ). Orthopaedic conditions were the most common condition recorded for initial hospital admissions with many of these for elective procedures and others resulting from falls. Over six months around $40 \%$ of the patients in the study were readmitted to hospital.

\section{Conclusions:}

Hospital admissions and re-admissions are the major health care cost in this cohort of older people. The costs of the Transition Care Program are higher than other community programs however are relatively small compared to the cost of an admission to hospital. Transition Care may have some impact on reducing hospital re-admissions and preventing or delaying residential care admissions and this could be further enhanced with streamlining admission processes to the Transition Care Program. 


\section{Introduction}

Governments are facing increasing pressures on health budgets with the proportion of GDP allocated to health continuing to rise in Australia (1). The ageing population is expected to be a key contributor to future increases in demand for health services, with estimates that health care spending will need to increase by $23 \%$ over the next two decades to meet the increased need for services for older Australians (2). Hospital spending is under particular scrutiny as these costs have risen at a far higher rate than any other sector of government spending (1). Australian research has shown that $25 \%$ of admissions for older people (aged over 70 years) were complicated by the inability to cope with activities of daily living (3) This has increased government interest in services that support older people to remain living in the community rather than being admitted to hospital or entering residential care prematurely. These support services have changed substantially over time with the development of the Home and Community Care Program, Community Aged Care Packages, and Extended Aged Care in the Home by the Australian Government. All contribute to the ability of older people to continue to manage at home (4). These services often require some time to implement and may not necessarily be suitable for the immediate post hospital period where extra assistance is commonly needed.

In response to this, specific post-hospital services for older people in the form of the Transition Care Program (TCP) were implemented in Australia in 2005. The program targets older patients at the conclusion of a hospital episode who have been assessed as eligible for at least low level residential aged care and who would benefit from support in a non-hospital environment to allow functional recovery (5). People usually receive the services in their own home; however, some enter a residential care transition care program prior to transitioning to the community or entering permanent residential care. The program provides services including home help and personal care (up to daily); slow stream rehabilitation including physiotherapy and occupational therapy (and sometimes other therapies and medical specialist services); nursing care and case management (6).The guidelines for TCP provide funding for an expected length of stay of eight weeks with a maximum period of 12 weeks post discharge from hospital (5) The aims are to improve or actively maintain the person's capacity for independent living, so that inappropriate extended hospital lengths of stay and premature admission to residential aged care are minimised. Patients are eligible to access the program from either public or private hospitals.

Previous research has demonstrated that both low and high needs recipients of TCP generally improve over the course of treatment and maintain that improvement for up to six months post discharge from hospital (7). Clinically meaningful improvements occur in both in functional capacity and in quality of life particularly in the domains of mobility, self care and usual activities $(7,8)$. Most recipients remain living in their own home (over 85\%) after the end of the TCP episode (7).

Despite growing concern in developed countries about ageing populations and the feared future strain on health care budgets, little has been achieved in maintaining health care costs within the bounds of natural growth in age demographics and inflation. Evidence that the 
resources used, particularly on post-acute health care for older people, represent value for money is lacking (9). Specific information, about what the drivers of costs for health care are, will enable better targeting of support programs like TCP to reduce the overall burden on the health system.

\section{Objective}

This paper aims to describe, from the perspective of the health care funder, the cost components of the Australian Transition Care Program. The paper also describes the health care resource use and costs for a group of Transition Care patients over a six month period.

\section{Methods}

The design was a prospective cohort observational study. All patients entering communitybased TCP at six sites in two states in Australia (Queensland and South Australia) were eligible to participate in the study. During an eleven month recruitment period from November 2009 to September 2010, 351 patients or their substitute decision maker gave consent to participate. Patients were followed up at six months post TCP admission to ascertain current living status. Information on the index hospital admission prior to TCP as well as readmissions to hospital over the six month follow-up period was obtained through administrative data on hospital separations from state health departments. Ethics approval was given by the relevant University Human Research Ethics Committee (HREC) as well as HRECs responsible for governance at each of the TCP sites.

A comprehensive profile of recipients' physical, mental and social functioning at admission and discharge was compiled (10). Resource use (comprising staff time, equipment, hospital care, residential care, medical and pharmaceutical use) was also documented. Transition care cost was based on staff time and equipment logs filled in by the teams on a daily basis. Staff time for each patient was identified as daily time spent on case management, nursing, allied health (physiotherapy, occupational therapy etc), personal care or home care. Direct patient time and indirect patient time (e.g. travel time, time with the patient or case management time) were multiplied by the relevant staff wage rate obtained from the South Australian and Queensland government wage rates, including on-costs, to calculate the costs of the service. Single use equipment (e.g. pads, wound dressings) were costed at the market price. Where the equipment was loaned, a rental rate was attributed to the patient based on the purchase cost of the item, depreciation rate and total life span of the equipment.

Following patient consent to release, pharmaceutical usage and Medicare items, (GP visits, allied health consultations) were collected from the Australian government Medicare and Pharmaceutical Benefits Scheme databases over six months following admission to TCP. The Medical Care collected in this database includes General Practitioner and Medical Specialist visits as well as Allied Health visits covered under Medicare Enhanced Primary Health Care items.

The cost of hospitalisation was calculated based on the Australian-Revised Diagnostic Related Groupings v5.2 (AR-DRG codes) provided with the hospital records obtained from Qld Health 
and SA Health. Cost weights (hospitalisation, average lengths of stay and costs of pharmacy items) were obtained from the National Hospital Cost Data Collection Round 13 (11) for the AR-DRG codes. Where AR-DRG codes were missing, an average cost per day of \$1131 per day was used based on the average daily cost of the four most frequent AR-DRG codes recorded at separation. These codes included rehabilitation (Z6o), hip and knee replacement (IO3-4) and other hip and femur procedure (IO8), representing $38 \%$ of separations. All other individual codes were less than $10 \%$ of separations with most codes only 1 or $2 \%$.

The cost of hospitalisation was adjusted to allow for variation from the average length of stay associated with the AR-DRG code. For patients who stayed under the average length of stay, the percentage of the total cost was used, e.g. $60 \%$ shorter stay results in a $60 \%$ lower than average hospital cost. For patients who stayed longer than the average length of stay, an additional daily cost of $\$ 629$ was used representing the average daily cost of AR-DRG Z6oA Factors influencing health status and other contacts with health services, the most common coding used for longer stays. For private hospital admissions, the costs of all pharmacy items were excluded from these calculations as these were included in the pharmaceutical benefits scheme data for private patients. Overnight and day re-admissions to hospital were analysed separately as day admissions occurred for planned reasons; primarily dialysis or day rehabilitation and most overnight re-admissions occurred for unplanned reasons.

For patients who entered community transition care following a residential care stay, the cost of the residential component of the patient's total care was estimated at $\$ 106.88$ per day based on daily government subsidy for a person of medium level of dependency with complex health care needs (12). Patient co-payments were excluded as the analysis is from a health services perspective. During the transition care period no other community services were received. While some patients received community services (for example help with housework and shopping) following the transition care period ( $\sim 2$ weeks post hospitalisation) these costs were not included in this analysis.

Total health care resource use was calculated for the six month follow up period by summing the hospital, medicare and pharmaceutical use, residential care and TCP costs. For this estimate, a cost of $\$ 12,433$ for an episode of TCP was used based on the cost per episode of care to the government (9) as the resource items collected during the study did not measure the total cost of providing the program.

Nonparametric descriptive statistics were calculated for the cost of TCP and the cost of other health care (hospital, pharmaceuticals and medical expenses). The primary diagnosis was evaluated by AR-DRG Code generated at hospital separation. This analysis was based on the four Queensland sites as AR-DRG codes were not provided for South Australian hospital admissions. A total of 260 AR-DRG codes related to the initial admission were analysed and presented descriptively by public or private setting. Cost and AR-DRG codes for re-admissions are also presented.

\section{Results}

Comprehensive details of the participants have been previously reported (7) In brief, the average age of the participants in the study was 79. Participants had an average of six recorded 
co-morbidities and were taking over nine medications. The individual cost components of TCP are presented in Table 1. Costs are presented as an average per patient receiving that particular service. The proportion (percentage) of the total cohort who receives each component (e.g. nursing, physio) is also provided. Expected values (average over the whole 351 participants) can be obtained by multiplying the mean cost by the proportion of the cohort receiving that intervention.

\section{$<$ INSERT TABLE 1 HERE $>$}

Health care consumed by the patients over a six month period is detailed in Table 2 . The majority of costs arose from the initial hospitalisation. Follow-up hospitalisation was also costly with around $40 \%$ of patients experiencing this event (table 2 ).

\section{$<$ INSERT TABLE 2 HERE>}

For the index hospitalisation, $43 \%(\mathrm{n}=111)$ of referrals to TCP were received from private hospitals. This figure is slightly above the national activity data that indicates that $40.6 \%$ of all separations occur in private hospitals (13).

Almost half of patients were admitted to TCP following a hospitalisation for an orthopaedic condition. Many of these were fractures with $37 \%$ of the cohort $(n=131)$ sustaining this injury, and almost half of these $(n=65)$ being hip fractures. Joint replacements accounted for $12.5 \%$ $(n=44)$ of the admissions, although some of these were hip replacements following fracture. The second most common AR-DRG code recorded in public hospitals was the category of Neurology; however, for private hospitals, rehabilitation - a descriptor falling into the "factors influencing health status and other contacts with health services" major grouping was the second most common code. The AR-DRG code for stroke represented $5 \%(n=18)$ of separations. All other categories were small with only one or a few patients in each.

Overnight re-admissions resulted in the second largest health care costs over six months for this cohort with a mean cost of $\$ 7,038$. A total of 142 patients re-hospitalised from the six sites. Re-admissions rates were $40.5 \%$ overall (up to six months post first hospital discharge) and varied between the six sites from 28 to $45 \%$ of all patients.

While the majority of initial hospital admissions leading to the transition care episode were for orthopaedic conditions, this was more rarely the cause of re-admission. The most common AR-DRG code for hospital re-admission was for rehabilitation with the second most common for circulatory system disorders. Syncope accounted for 11 separations and heart failure a further 11 separations. For the musculoskeletal disorders, joint replacements accounted for seven of the total, and hip fracture for two. Stroke accounted for three of the nervous system category. 
Day admissions were also common with the primary reasons for day admission being dialysis (151 occasions of service), cancer treatment (34 occasions) and for day rehabilitation (32 occasions).

By the six month follow up, 16 patients (4.6\%) had been admitted to permanent residential aged care. At an average stay of three months, these patients consumed an average of $\$ 9,833$ per person in government subsidies with total costs of over $\$ 150,000$ to the government.

\section{Discussion}

The resource use collected in this study of TCP represented almost 11 hours per week of direct patient care. This was spread reasonably evenly across the different health professionals and care workers in the TCP service. Costs not estimated in this study include fixed costs such as buildings required to house teams and ongoing costs such as cars required for a community team, and staff time for team meetings, training and for sick and recreational leave. The cost of TCP is relatively high compared to other community care packages. Given a seven week package, the average daily cost of TCP is estimated at $\$ 250$, which is considerably higher than both the Community Aged Care Package (for low level care) daily subsidy of \$37.32, which provides around five hours per week input, and for the Extended Aged Care at Home (for high level care needs) of $\$ 124.75$, which provides about 15 hours per week. (14)

We have previously demonstrated that patients do show improved quality of life by the end of transition care and maintain this over six months (15) and there is some evidence that more intense intervention (as provided in TCP compared to other community care packages) does have a greater effect on improving functional outcomes(16). However, it is unclear whether the higher cost of this program compared to other community support packages is worthwhile as there is no data on which to make a direct comparison.

Whilst the program is primarily funded federally, the teams are established and managed by state government health authorities. This leads to systematic differences in the type and amount of services provided. Even within states, differences are evident with some teams providing all services from within the team and other outsourcing particular components such as home care services or allied health services. These differences may be positive in that the program is able to flexibly fill gaps in local services. However, the differential re-admission rates between sites may reflect different practices and types of services given.

The largest health care costs in this cohort were firstly, the initial hospitalisation and secondly, re-admissions to hospital. Reducing costs in the initial hospitalisation could be achieved with better and faster referral pathways and more responsive assessments of long stay patients to enable faster access to TCP. The re-admission rate is high; however, this is a high risk population. The rate of readmission is lower than that recorded in the 2008 report on transition care that reported $51 \%$ of community TCP recipients were readmitted to hospital nationally (46\% and $48 \%$ rate in QLD and SA respectively) as compared to $40.5 \%$ in this study(16). The rates of admission to residential care were slightly lower, with the previous evaluation recording $7 \%$ nationally ( $5 \%$ and $6 \%$ in QLD and SA) by six months as compared to $4.6 \%$ in this study (16). This may reflect that transition care has matured over time and is 
having a larger effect on both readmission rates and residential care admissions. Readmission may occur with no discussion with the TCP provider. A more structured primary care approach with involvement of the TCP provider in decision making may enable more resources to be directed to the patient in order to enable them to avoid hospital admission.

Costs of health care in this cohort of older people were primarily driven by long hospital stays from orthopaedic and musculoskeletal conditions and these included elective procedures. Differences may exist in screening of individuals likely to benefit or not from elective procedures between different hospitals and different funding models. Providing individualised cost-effectiveness information has been suggested as a method to help inform health care decisions for clinicians and their patients to improve costs and outcomes in the health care system (17).

A large proportion of the cohort (37\%) had orthopaedic procedures relating to fractures with most involving the hip. More widespread adoption of proven effective falls and fracture prevention programs for the older population and ensuring osteoporosis in managed effectively in the community may mitigate the growth of these costs in the future.

A review of Australian community packages for older people noted that the delivery of programs is often fragmented and uncoordinated with many small providers delivering services (18). Whether the delivery of TCP would be improved by central administration or by provision by non government organisations should be considered. Regardless; a more seamless approach to integrating other community services after the TCP episode of care is required.

Reducing the cost of providing care to this group of older frail people primarily needs to focus on reducing hospital stays and managing older people in the community. Regardless of whether the extra cost of providing the TCP as opposed to other community packages is justifiable, an integrated approach to the management of older people in the community is needed. The current minefield of federal, state and NGO provision of services with no consistency within cities, states or the nation, leaves older people and their primary practitioners with a confusing array of services with gaps, overlaps and limited information on what is provided where, for whom, and at what time. These anomalies have been recognised federally and the Living Longer Living Better aged care reform package announced in April 2012 (19) aims to address inequities and build a fairer, more consistent aged care system. Only time will determine if these reforms go far enough.

\section{Limitations}

The observational nature of this study and lack of a comparison group means that the effect of TCP on relevant outcomes such as re-admissions and length of hospital stay cannot be determined. The study sites were limited to two Australian states and, since patterns of provision of TCP vary considerably between states, the results may not be generalizable to all TCP recipients (16).

The sample size of this study is small when considering cost outcomes that are highly skewed in nature. This limits the precision of the estimates given for the health care costs particularly 
for re-admissions where fewer than half experienced this event. However, a strength of the study was that extensive cost and health resources use data were collected, allowing costs to be attributed at an individual patient level.

\section{Conclusion}

Hospital admissions and re-admissions are the major health care cost in this cohort of older people. The costs of TCP are relatively small compared to the cost of an admission to hospital. Reducing further health care costs in this cohort would require improved co-ordination of services to allow faster entry into TCP. 


\section{References}

1. Daley J, McGannon C, Savage J. Budget pressures on Australian governments. Canberra: Grattan Institue 2013.

2. Australian Institute of Health and Welfare. Australia's health 2012, . Canberra: AIHW; 2012.

3. Kingston M. Determining the professional attributes of a hospitalist: experience in one Australian metropolitan hospital. Intern Med J. 2005 May;35(5):305-8. PubMed PMID: 15845116.

4. Bruen W. Aged care in Australia: past, present and future. Australasian Journal on Ageing. 2005;24(3):130-3.

5. Australian Government Department of Health and Ageing. Transition Care Program Guidelines. Canberra, ACT: Australian Government; 2011.

6. Gray L, Travers CM, Bartlett HP, Crotty M, Cameron ID. Transition care: will it deliver? Med J Aust. 2008 Feb 18;188(4):251-3. PubMed PMID: 18279139. eng.

7. Peel NM, Hubbard RE, Gray LC. Impact of post-acute transition care for frail older people: a prospective study. JFrailty Aging. 2013;2(3):165-71.

8. Comans TA, Peel NM, Gray LC, Scuffham PA. Quality of life of older frail persons receiving a post-discharge program. Health Qual Life Outcomes. 2013;11:58. PubMed PMID: 23587460. Epub 2013/o4/17. eng.

9. Hall CJ, Peel NM, Comans TA, Gray LC, Scuffham PA. Can post-acute care programmes for older people reduce overall costs in the health system? A case study using the Australian Transition Care Programme. Health Soc Care Community. 2012;20(1):97-102.

10. Peel NM, Hubbard RE, Gray LC. Impact of post-acute transition care for frail older people: a prospective study Journal of Frailty and Aging. 2013;[EPub ahead of print 30/1/2013]. 11. Department of Health and Ageing. Cost Report Round 13 (2008-2009). Canberra ACT: Department of Health and Ageing, 2009.

12. Australian Government Department of Health and Ageing. Australian Government Subsidies and Supplements Canberra2013 [cited 201312 April]. Available from: http://www.health.gov.au/internet/main/publishing.nsf/content/ageing-subs-suppcurrent.htm.

13. Australian Institute of Health and Welfare. Australian hospital statistics 2009-10. Health services series no. 40. Cat. no. HSE 107. . Canberra: AIHW; 2011.

14. Australian Government Department of Health and Ageing. Home-based care 2012 [cited 2012 10 October]. Available from:

http://www.health.gov.au/internet/main/publishing.nsf/Content/ageing-commcarecomcprov-ccpindex.htm.

15. Comans TA, Peel NM, Gray LC, Scuffham PA. Quality of life of older frail persons receiving a post-discharge program. Health and Quality of Life Outcomes. 2013;11(1):58.

16. Flinders Consulting. National Evaluation of the Transition Care Program: Final Report. Canberra, ACT: Department of Health and Ageing; 2008.

17. Ioannidis JP, Garber AM. Individualized cost-effectiveness analysis. PLoS Med. 2011 Jul;8(7):e10o1058. PubMed PMID: 21765810. Pubmed Central PMCID: 3134464.

18. Henderson EJ, Caplan GA. Home Sweet Home? Community Care for Older People in Australia. Journal of the American Medical Directors Association. 2008;9(2):88-94.

19. Australian Government Department of Health and Ageing. Living Longer Living Better - Aged Care Reform in Action Canberrazo12 [cited 201316 May]. Available from:

http://www.livinglongerlivingbetter.gov.au/. 
Tables

Table 1 Transition Care Program components of care (proportion receiving service and costs per patient)

\begin{tabular}{|c|c|c|c|c|c|c|c|c|c|}
\hline & $\begin{array}{l}\text { Case } \\
\text { Management }\end{array}$ & Nursing & Physiotherapy & $\begin{array}{l}\text { Occupational } \\
\text { Therapy }\end{array}$ & Home Care & $\begin{array}{l}\text { Personal } \\
\text { Care }\end{array}$ & $\begin{array}{l}\text { Other Allied } \\
\text { Health* }\end{array}$ & Equipment & Travel \\
\hline $\begin{array}{l}\text { Patients receiving } \\
\text { service (\%) }\end{array}$ & $100 \%$ & $78 \%$ & $92 \%$ & $88 \%$ & $38 \%$ & $88 \%$ & $64 \%$ & $57 \%$ & $85 \%$ \\
\hline Mean (sd) & $\begin{array}{l}\$ 626.50 \\
(\$ 632.92)\end{array}$ & $\begin{array}{l}\$ 516.73 \\
(\$ 553.78)\end{array}$ & $\begin{array}{l}\$ 431.07 \\
(\$ 417.99)\end{array}$ & $\begin{array}{l}\$ 642.70 \\
(\$ 732.20)\end{array}$ & $\begin{array}{l}\$ 488.47 \\
(\$ 580.41)\end{array}$ & $\begin{array}{l}\$ 815.28 \\
(\$ 858.15)\end{array}$ & $\begin{array}{l}\$ 443.76 \\
(\$ 616.58)\end{array}$ & $\begin{array}{l}\$ 536.71 \\
(\$ 510.37)\end{array}$ & $\begin{array}{l}\$ 751.40 \\
(\$ 594.38)\end{array}$ \\
\hline Median (IQR) & $\begin{array}{l}\$ 461.04 \\
(\$ 191.25 \\
\$ 823.94)\end{array}$ & $\begin{array}{l}\$ 372.16 \\
(\$ 183.30 \\
\$ 644.59)\end{array}$ & $\begin{array}{l}\$ 309.01 \\
(\$ 174.43 \\
\$ 529.73)\end{array}$ & $\begin{array}{l}\$ 404.09 \\
(\$ 183.37 \\
\$ 923.63)\end{array}$ & $\begin{array}{l}\$ 290.73 \\
(\$ 149.73 \\
\$ 621.39)\end{array}$ & $\begin{array}{l}\$ 498.85 \\
(\$ 195.98 \\
\$ 1111.76)\end{array}$ & $\begin{array}{l}\$ 225.87 \\
(\$ 63.63, \\
\$ 547.57)\end{array}$ & $\begin{array}{l}\$ 377.60 \\
(\$ 165.0 \\
\$ 766.10)\end{array}$ & $\begin{array}{l}\$ 623.32 \\
(\$ 350.95, \\
\$ 967.23)\end{array}$ \\
\hline
\end{tabular}

${ }^{*}$ Other Allied Health includes Speech Pathology, Dietetics, Podiatry, Social Work and Allied Health Assistant time

Table 2 Health care resources used over six months (cost per patient)

\begin{tabular}{|c|c|c|c|c|c|c|c|c|c|}
\hline & $\begin{array}{l}\text { Index } \\
\text { hospitalisation }\end{array}$ & $\begin{array}{l}\text { Follow up } \\
\text { hospitalisation }\end{array}$ & $\begin{array}{l}\text { Follow up day } \\
\text { hospital }\end{array}$ & $\begin{array}{l}\text { Medical } \\
\text { Services }\end{array}$ & $\begin{array}{l}\text { Pharmaceu } \\
\text { ticals }\end{array}$ & $\begin{array}{l}\text { Residential } \\
\text { TCP }\end{array}$ & $\begin{array}{l}\text { Communit } \\
\text { y TCP }\end{array}$ & $\begin{array}{l}\text { Permanent } \\
\text { residential } \\
\text { care }\end{array}$ & $\begin{array}{l}\text { Total health } \\
\text { care cost * }\end{array}$ \\
\hline $\begin{array}{l}\text { Mean } \\
\text { (sd) }\end{array}$ & $\begin{array}{l}\$ 39,516 \\
(\$ 46,939)\end{array}$ & $\begin{array}{l}\$ 7,038 \\
(\$ 16,001)\end{array}$ & $\$ 919(\$ 4,363)$ & $\begin{array}{l}\$ 1,798 \\
(\$ 2,345)\end{array}$ & $\begin{array}{l}\$ 1,024 \\
(\$ 1,024)\end{array}$ & $\begin{array}{l}\$ 642 \\
(\$ 2,085)\end{array}$ & $\begin{array}{l}\$ 4,519 \\
(\$ 2,887)\end{array}$ & $\$ 448.23$ & $\begin{array}{l}\$ 62,499 \\
(\$ 44,853) *\end{array}$ \\
\hline $\begin{array}{l}\text { Median } \\
\text { (IQR) }\end{array}$ & $\begin{array}{l}\$ 26,405 \\
(\$ 16,481 \\
\$ 43,340)\end{array}$ & $\$ 0(\$ 0, \$ 3,749)$ & $\$ 0(\$ 0, \$ 0)$ & $\begin{array}{l}\$ 1,162 \\
(\$ 613 \\
\$ 1,798)\end{array}$ & $\begin{array}{l}\$ 839(\$ 381 \\
\$ 1,240)\end{array}$ & $0(\$ 0, \$ 0)$ & $\begin{array}{l}\$ 3,946 \\
(\$ 2,603 \\
\$ 5,579)\end{array}$ & $\$ 448.23$ & $\begin{array}{l}\$ 48,557 \\
(\$ 35,393 \\
\$ 72,595)\end{array}$ \\
\hline
\end{tabular}

TCP = transition care program; IQR=Interquartile Range 
*Total cost of health care is calculated with a cost of $\$ 12,433$ for the transition care program representing the actual cost to government of providing the service per person 